\title{
PAPER
}

\section{Head injury outcome prediction in the emergency department: a role for protein S-100B?}

\author{
W J Townend, M J Guy, M A Pani, B Martin, D W Yates
}

J Neurol Neurosurg Psychiatry 2002;73:542-546

See end of article for authors' affiliations

.....................

Correspondence to:

Dr W Townend,

Emergency Department,

Hope University Hospital,

Salford M6 8HD, UK ;

wtownend@

fs l.ho.man.ac.uk

Received 25 September 2001

In revised form 10 July 2002

Accepted 30 July 2002

\begin{abstract}
Background: Biochemical markers released after head injury may reflect the degree of brain damage, which is related to subsequent disability. If the serum level of a marker were found to be related to outcome, then earlier identification and intervention would be possible.

Objective: To investigate the potential of the serum marker S-100B protein to predict the outcome after head injury.

Methods: Blood samples for S-100B concentrations were taken from 148 adults within six hours of a head injury (initial Glasgow coma score 4-15). Patients were recruited from the emergency departments of four hospitals in Greater Manchester, United Kingdom. Outcome was assessed in 119 patients $(80 \%)$ at one month using the extended Glasgow outcome scale (GOSE).

Results: A significant inverse correlation between serum S-100B level and GOSE was found (Spearman's $\rho=-0.349, p<0.0001)$. A serum S-100B concentration of $>0.32 \mu g / l$ predicted severe disability (GOSE < 5) at one month with a sensitivity of $93 \%(95 \%$ confidence interval $68 \%$ to $100 \%)$, a specificity of $72 \%$ (54\% to $79 \%$ ), and a negative predictive value of $99 \%$ (93\% to $100 \%)$.

Conclusion: Serum S-100B concentration can be used in the emergency department to identify patients with head injury who are most likely to have a poor outcome at one month.
\end{abstract}

O ne million head injured patients present to the emergency departments of the United Kingdom annually. ${ }^{1}$ Head injury is the commonest cause of death from trauma in the United Kingdom, with about 5000 fatalities a year. ${ }^{2}$ Thornhill et al showed that a significant number of survivors are disabled by their injuries. ${ }^{3}$ They found that outcome following head injury did not appear to depend on the apparent initial severity. From a cohort of 2962 young people and adults in their study, $48 \%$ of those with severe head injury (Glasgow coma score (GCS) of 8 or less), $45 \%$ with moderate head injury (GCS 9-12), and 47\% with mild injury (GCS 13-15) were disabled 12 months later.

Most emergency management protocols are focused exclusively on the identification of the relatively small number of patients who may require operative intervention, as early surgery is believed to improve outcome. However, early rehabilitation can also be effective for the much larger number of patients who do not require surgery. ${ }^{4}$ Clearly it would be neither possible nor appropriate to offer such care to every patient with mild or moderate head injury. However, the early identification of patients more likely to experience prolonged disabling symptoms would allow rehabilitation services to be used more effectively.

S-100B has been investigated as a possible marker of brain injury in recent years. It is a calcium binding protein that is highly expressed in the astroglial cells of brain tissue. It is known to cross the blood-brain barrier in measurable quantities after head injuries, even those classified as mild. ${ }^{56}$ Raabe et al found an inverse relation between serum S100-B level and outcome following severe head injury ${ }^{7}$; Ingebrigtsen et al found that patients with raised serum S-100B concentration following head injury required a longer period of inpatient observation ; and Herrmann et al showed that a raised serum S-100B concentration was associated with a worse neuropsychological outcome six months after head injury. ${ }^{9}$

Our aim in the present study was to evaluate serum S-100B concentration as a possible diagnostic test of outcome that could be used in the emergency department.

\section{METHODS}

Approval was obtained from the local research ethics committee for this prospective study. Patients were recruited from February 2000 for seven months.

\section{Participants}

We recruited 148 adult patients. Inclusion criteria were head injury and presentation to the emergency department within six hours of injury. The definition of head injury used for this study was "any blow to the head causing a clinical diagnosis of head injury to be made, even if insufficient to cause definite loss of consciousness." ${ }^{\prime 10}$ There were no specific exclusion criteria. In particular there was no upper age limit, and patients with concurrent injuries to other body regions were included. Those with suspected alcohol or drug ingestion and with pre-injury morbidity were also included. A six hour cut off was chosen as it is thought that S-100B is rapidly cleared from the serum, with a half life estimated at around two hours, " although definitive clearance characteristics following head injury have yet to be established. Initial injury severity was assessed using the Glasgow coma score. ${ }^{12}$ All other investigations and treatments were in accordance with current departmental guidelines.

\section{Setting}

Patients were recruited from the emergency departments of four hospitals in Greater Manchester, two of which are teaching hospital departments and two are within district general hospitals.

\section{Data collection}

Demographic data were collected on each participant, and the time and mechanism of injury recorded. Relevant symptoms and signs were also recorded, along with radiological investigations and information on patient disposal.

\section{S-100B assay}

A venous blood sample was taken from each patient and the time of collection recorded. Blood samples were refrigerated 


\begin{tabular}{|c|c|}
\hline $\begin{array}{l}\text { GOSE } \\
\text { score }\end{array}$ & Performance level \\
\hline 1 & Dead \\
\hline 2 & Vegetative state \\
\hline 3 & Lower severe disability: completely dependent on others \\
\hline 4 & $\begin{array}{l}\text { Upper severe disability: dependent on others for some } \\
\text { activities }\end{array}$ \\
\hline 5 & $\begin{array}{l}\text { Lower moderate disability: unable to return to work or } \\
\text { participate in social activities }\end{array}$ \\
\hline 6 & $\begin{array}{l}\text { Upper moderate disability: return to work at reduced } \\
\text { capacity, reduced participation in social activities }\end{array}$ \\
\hline 7 & $\begin{array}{l}\text { Lower good recovery: good recovery with minor social or } \\
\text { mental deficits }\end{array}$ \\
\hline 8 & Upper good recovery \\
\hline
\end{tabular}

and transferred to the laboratory within 48 hours. The stability of S-100B concentrations in whole blood for this time period has been demonstrated. ${ }^{13}$ Samples were centrifuged and the serum was frozen at $-20^{\circ} \mathrm{C}$ until analysed. S-100B concentration was measured in duplicate on each patient using the commercially available LIA-mat $\AA$ Sangtec $(\AA 100$ kit (Cambridge Life Sciences, Cambridge, United Kingdom). The minimum measurable S-100B value for this assay is $0.02 \mu \mathrm{g} / \mathrm{l}$.

\section{Outcome measures}

An assessment of neurological disability was made at one month using the extended Glasgow outcome score (GOSE) ${ }^{14}$ (table 1). This is an amendment to the original, widely used Glasgow outcome scale. ${ }^{15}$ It is an eight point scoring system and was administered in this study as a standardised interview. ${ }^{16}$ The Glasgow outcome scale is the most widely used method describing the overall outcome after head injury, and its extended form (GOSE) has been validated with regard to other outcome measures including subjective reports of health outcome such as the short form 36 assessment ${ }^{17}$ and neuropsychological tests. ${ }^{18}$

Patients and their relatives were contacted by telephone, as in the cohort study by Thornhill et al on a similar population. ${ }^{3}$ Outcome was assessed blind to the serum S-100B level. A GOSE score between 5 and 7 denotes moderate disability and encompasses individuals with significant restrictions in lifestyle or work capacity, or both. Those assigned a score of 4 or less (severe disability) are unable to support themselves for 24 hours in the community. ${ }^{14}$ Patients with previous morbidity were assessed for any change associated with their head injury and assigned a good outcome if able to function at the same level as before the injury.

\section{Statistical analysis}

The correlation between clinical variables measured at initial assessment and GOSE score was assessed using Spearman's method for non-normal data. The extent to which the S-100B levels differed between those disabled and those with a good recovery after injury was assessed using receiver operator characteristics (ROC) plots. The ROC plot is obtained by calculating the sensitivity and specificity for every distinct observed data value, and plotting sensitivity against 1-specificity. Diagnostic accuracy is given by the area under the curve. This area represents the probability that a person, chosen at random, with disability after head injury has a higher S-100B level than a randomly chosen, head injured person without disability. ${ }^{19}$ To assess the accuracy of S-100B estimation in identifying any significant disability after head injury, outcome was divided into good, with a GOSE score of 7 or 8

Table 2 Demographic data

\begin{tabular}{lllll}
\hline Variable & Total $(n=148)$ & $\begin{array}{l}\text { Followed up } \\
(n=119)\end{array}$ & Not followed up $(n=29)$ & $p$ Value \\
\hline Age (mean (SD)) & $47.4(20.9)$ & $49.0(21.2)$ & $39.8(17.7)$ & 0.06 \\
Male & $93(63 \%)$ & $71(60 \%)$ & $22(76 \%)$ & 0.33 \\
Initial GCS 15 & $130(88 \%)$ & $103(87 \%)$ & $27(93 \%)$ & 0.60 \\
LOC & $52(35 \%)$ & $43(36 \%)$ & $9(31 \%)$ & 0.57 \\
Discharged from ED & $93(63 \%)$ & $73(61 \%)$ & $20(69 \%)$ & 0.73 \\
\hline
\end{tabular}

ED, emergency department; GCS, Glasgow coma score; LOC, loss of consciousness.

Table 3 Radiological findings, initial Glasgow coma score, S-100B level, and extended Glasgow outcome scale in 15 patients undergoing computed tomography of the head as part of routine evaluation

\begin{tabular}{lllll}
\hline $\begin{array}{l}\text { Patient } \\
\text { No }\end{array}$ & GCS & CT findings & S100B & GOSE \\
\hline 1 & 15 & Normal & 0.30 & 6 \\
2 & 15 & Basal skull fracture only & 0.49 & 3 \\
3 & 15 & Skull fracture only & 0.51 & 8 \\
4 & 15 & Normal (skull fracture seen on x ray) & 0.18 & 8 \\
5 & 14 & Basal skull fracture; intracerebral and subdural blood & 4.56 & 1 \\
6 & 14 & Small amount subdural blood & 2.57 & 5 \\
7 & 14 & Normal & 0.82 & 3 \\
8 & 14 & Basal skull fracture only & 2.15 & 8 \\
9 & 14 & Intracerebral blood with raised intracranial pressure & 1.00 & 4 \\
10 & 11 & Basal skull fracture with subarachnoid blood & 0.35 & 3 \\
11 & 8 & Normal & 0.05 & 8 \\
12 & 7 & Small subdural with contusion & 4.8 & 1 \\
13 & 7 & Intracerebral blood with raised intracranial pressure & 2.32 & 2 \\
14 & 7 & Chronic multi-infarct atrophy. No acute changes & 6.7 & 1 \\
15 & 4 & Depressed fracture; extensive haemorrhagic contusions, & 18.5 & 1 \\
\multicolumn{7}{l}{ subarachnoid blood } & & \\
\hline CT, computed tomography; GCS, Glasgow coma score at initial assessment; GOSE, Glasgow outcome \\
scale, extended.
\end{tabular}




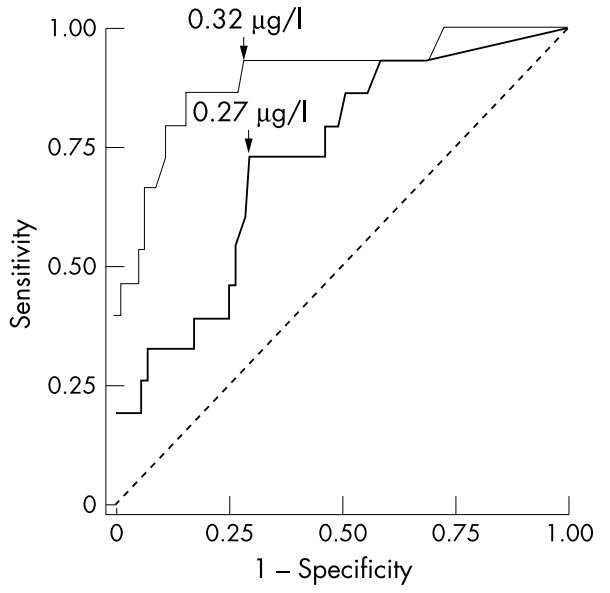

Figure 1 Receiver operator characteristics curves of S-100B protein concentration for neurological outcome at one month. Bold line: moderate disability (GOSE < 7); area under the curve $=0.770$ (95\% confidence interval 0.67 to $0.87 ; p<0.001)$. Narrow line: severe disability (GOSE $<5)$; area under the curve $=0.889(0.792$ to $0.985 ; p<0.0011$. GOSE, Glasgow outcome scale, extended.

and adverse, with a score of less than 7. The subgroup of patients with the most severe limitations-those with GOSE $<5$-were also of interest, so analysis was repeated with outcome dichotomised at that level. ROC curves were therefore plotted with an adverse outcome defined as GOSE $<7$ and as GOSE $<5$. The outcome data from the subgroup with an initial GCS of 13-15 were also analysed in this way, as such patients are customarily grouped together as mildly injured.

The ROC plots were used to identify cut off points that ensured a high proportion of patients disabled after injury would be detected (high sensitivity) at the cost of a relatively high false positive rate (low specificity). Analysis was performed using the Statistical Package for Social Sciences (SPSS).

\section{RESULTS}

\section{Follow up}

We recruited 148 patients ranging in age from 18 to 94 years. GOSE scores were assigned to 119 of these (80\%). Follow up was attempted in all patients. Those not assigned GOSE scores had all been discharged back to the community following their injuries and could not be contacted by telephone or mail. None of this group had died or undergone operative procedure at the regional neurosurgical centre within the follow up period. Interviews were performed by WJT in 105 cases and by $\mathrm{BM}$ in 14.

Comparison of the demographic data and injury characteristics of those reviewed and those lost to follow up are summarised in table 2 . The groups were assessed by logistic regression analysis. There was a trend towards a less successful follow up of younger patients, but no statistically significant differences were found.

\section{Clinical findings and outcome assessment}

Most patients were classified as having sustained mild injuries (GCS 13-15) (113 of 119; 95\%) and the majority (90 of 119; $76 \%$ ) were considered to have made a good recovery at follow up (GOSE > 6). There was a significant correlation between initial GCS and GOSE (Spearman's $\rho=0.350, p<0.001$ ). However, within the subgroup of those patients presenting with GCS 13-15 a good recovery was not assured. A GOSE score of less than 7 was found in 24 of 113 of this mild injury group $(21 \%)$.

Sex, age over 50, presence of post-traumatic amnesia, loss of consciousness, and initial GCS less than 14 were entered into
Table 4 Diagnostic characteristics of S-100B concentration as a test for moderate disability one month after head injury

\begin{tabular}{llll}
\hline S-100B & GOSE $<7$ & GOSE $\geqslant 7$ & Total \\
\hline$\geqslant 0.27 \mu \mathrm{g} / \mathrm{l}$ & True positive 22 & False positive 28 & 50 \\
$<0.27 \mu \mathrm{g} / \mathrm{l}$ & False negative 7 & True negative 61 & 68 \\
Total & 29 & 89 & 118 \\
\hline
\end{tabular}

Sensitivity $=22 / 29=76 \%(95 \% \mathrm{Cl}, 56 \%$ to $90 \%)$.

Specificity $=61 / 89=69 \%(95 \% \mathrm{Cl}, 58 \%$ to $78 \%)$

Negative predictive value $=61 / 68=90 \%(95 \% \mathrm{Cl}, 79 \%$ to $96 \%)$

$\mathrm{Cl}$, confidence interval; GOSE, Glasgow outcome scale, extended.

Table 5 Diagnostic characteristics of S-100B concentration as a test for severe disability one month after injury

\begin{tabular}{llll}
\hline S-100B & GOSE $\leqslant 4$ & GOSE $>4$ & Total \\
\hline$\geqslant 0.32 \mu \mathrm{g} / \mathrm{I}$ & True positive 14 & False positive 29 & 43 \\
$<0.32 \mu \mathrm{g} / \mathrm{I}$ & False negative 1 & True negative 74 & 75 \\
Total & 15 & 103 & 118 \\
\hline
\end{tabular}

Sensitivity $=14 / 15=93 \%(95 \% \mathrm{Cl}, 68 \%$ to $100 \%)$.

Specificity $=74 / 103=72 \%(95 \% \mathrm{Cl}, 62 \%$ to $80 \%)$

Negative predictive value $=74 / 75=99 \%(95 \% \mathrm{Cl}, 93 \%$ to $100 \%)$

$\mathrm{Cl}$, confidence interval; GOSE, Glasgow outcome scale, extended.

a logistic regression model. None of these factors predicted outcome independently.

Fifteen patients underwent cranial computed tomography (CT) following injury. These findings are shown in table 3.

\section{S-100B and outcome}

The serum concentration of S-100B was estimated for patients with known GOSE scores. The median time of sampling was 130 minutes after injury (range 18 to 360 minutes).

One blood sample was misplaced and so analysis was performed on data from 118 patients. S-100B was detectable in the serum in 92 of 118 patients $(78 \%)$. One blood sample was misplaced and so analysis was performed on data from 118 patients. A significant inverse correlation between S-100B level and GOSE score was found (Spearman's $\rho=-0.349$, $\mathrm{p}<0.001)$. When added to the logistic regression model, only serum S-100B level independently predicted outcome $(r=-0.13, \mathrm{p}=0.039)$.

Receiver operator characteristics (ROC) curves were plotted (fig 1). Cut off points were chosen on each of the curves that would ensure the detection of the largest proportion of those disabled with the least compromise of specificity (fig 1). These were $0.27 \mu \mathrm{g} / \mathrm{l}$ for moderate disability and $0.32 \mu \mathrm{g} / \mathrm{l}$ for severe disability. The diagnostic characteristics of these cut off points are summarised in tables 4 and 5 .

\section{Mild head injury outcome assessment}

Data analysis was repeated for the subgroup with initial GCS 13-15 $(\mathrm{n}=112)$. A cut off point of $0.48 \mu \mathrm{g} / \mathrm{l}$ was shown to predict a GOSE score of $<5$ with a sensitivity of $90 \%(95 \%$ confidence interval $55 \%$ to $99 \%$ ), a specificity of $83 \%$ (75\% to $90 \%$ ), and a negative predictive value of $99 \%$ (94\% to $100 \%$ ).

\section{DISCUSSION}

In this study we evaluated the potential role of S-100B as an early predictive marker of disability in adult patients with head injuries. Our results confirm that recovery for some patients with mild brain injury and no need for surgery is neither prompt nor complete. ${ }^{3}$ The disability rate (GOSE $<7$ ) in this heterogeneous cohort was 29 of 119 (24\%), with 15 of 119 $(13 \%)$ severely disabled (GOSE $<5)$. This rate is lower than in the most recent, large scale study of a similar population. ${ }^{3}$ This 
difference might be accounted for by our inclusion of patients with less severe injuries. We included those whose head injury was not associated with loss of consciousness or amnesia and those discharged directly from the emergency department.

\section{Outcome assessment}

Adults presenting to emergency departments with "minor" head injuries are difficult to follow up for research purposes. ${ }^{4}$ We aimed to minimise this difficulty by assessing outcome earlier than is customary, at one month. This approach was vindicated by our follow up rate of $80 \%$, which compares favourably with previous work (41\% to $71 \%) .{ }^{3}{ }^{4}$ A one month follow up was described as the earliest time point for reasonable outcome assessment in the review that accompanied the publication of the standardised GOSE interview used in our study. ${ }^{20}$

It was anticipated that those fully recovered at one month would remain well and would therefore require no further input. This is supported by a study of patients with head injury and extracranially injured controls followed up at three and six months using the GOSE by Levin et al, which showed deterioration with time to be extremely unlikely. ${ }^{18}$ Most patients in our study had mild injuries and were either discharged directly from the emergency department or following an inpatient stay of less than 48 hours. In this group the one month interval seemed particularly appropriate in determining which patients had recovered. If the aim of S-100B estimation is to discharge those with low levels from further intervention knowing that a good recovery is extremely likely, then this group is of interest.

It is acknowledged that for the more severely disabled the one month follow up interval does not truly reflect "outcome" as much as poor progress. A proportion of those severely disabled at one month will recover fully, as improvement with time has been demonstrated. ${ }^{21}$ Nonetheless, some of those with problems at one month in this study are clearly at risk of chronic morbidity, and the ability to identify them at one month would allow early intervention to be offered.

A GOSE of $>6$ denotes a return to full activity and no need for further support from rehabilitation services. Outcome was dichotomised at this level for that reason. It is unlikely that many patients who develop non-surgical complications will currently be identified or treated, as a postal survey in 1994 revealed that $87 \%$ of emergency departments in England and Wales offered no formal follow up to head injured patients. ${ }^{22}$ Resources to set up a service offering such care are likely to be limited, so identification of those with most to gain from treatment would also be important. We therefore assessed the accuracy with which S-100B identified more severe impairment-that is, a GOSE score of 4 or less.

\section{S-100B in those lost to follow up}

We did not measure the S-100B concentrations in patients who were not followed up, to minimise cost. We considered those whose levels we did measure to be representative of the whole group, based on demographic data and initial clinical findings. We were also satisfied that our data were not biased by loss to follow up because of death or readmission to hospital. This approach, however, does not allow us to assess whether the association between S-100B level and outcome differs for those we could not contact. We know, however, that the outcome for those lost to follow up is likely to have been favourable, and so there could not have been a large proportion of false negative results in this group. We therefore conclude that the results presented are not biased towards overestimating the sensitivity of the test. We cannot exclude a high false positive rate among those not followed up.

Identification of severe and moderate disability

In our cohort, S-100B estimation was a better detector of severe disability than of moderate disability. One explanation of such an effect is the lesser impact of confounding factorssuch as premorbid personality or the mechanism of injury-at more severe levels of disability. At the upper levels of the GOSE, scores relate to more subtle interpretations of how symptoms affect function after head injury, such as the impact on personal relationships. ${ }^{16}$ In this context, an assault might be expected to have a bigger psychological impact than a simple fall, regardless of the extent of brain tissue damage. This effect would clearly not apply to those who have died, and would be expected to be of less importance to those unable to function independently. Therefore outcome would be reflected more accurately by a measure of brain injury in more severely disabled individuals. A larger cohort of patients would be needed to investigate such an effect, however. The cut off point for severe disability was higher than that for moderate disability, reflecting the significant inverse correlation between S-100B level and GOSE that was found.

\section{S-100B and CT scan results}

It has been suggested that S-100B might have potential as a screening tool for the requirement for cranial CT following head injury. ${ }^{23}$ This is supported by our data. The only patient with a low S-100B among those imaged had a normal scan, despite a GCS of 8 at initial assessment. In contrast, S-100B concentrations were raised in two patients without radiological evidence of brain injury on CT who had not recovered at one month. According to these data, however, not all patients with severe disability at one month undergo CT scanning when current United Kingdom guidelines are followed. These findings require confirmation in a larger cohort.

\section{S-100B and extracranial injury}

Recent data suggest that S-100B measured in the serum might partly be derived from extracranial sources in the multiply injured patient. ${ }^{24}$ We included patients with extracranial injury in an attempt to assess S-100B across the full spectrum of head injury presentation and to reflect the normal clinical situation in an emergency department. An extracranial source of S-100B might offer one explanation for the relatively high false positive rate we found.

We did not investigate the potential confounding effect of an extracranial source of S-100B in the present study. Recruiting comparable controls was not thought possible because of the heterogeneity of our patients with regard to both the mechanism and the severity of their injuries. A comparable control group would include patients with multiple extracranial injuries who could have a greater degree of injury to the brain through deceleration than those with isolated trauma to the head, even if a history of percussive trauma to the cranium was absent. It would be important to consider this effect in any further data from traumatised control patients.

Confounding by extracranial S-100B release would be of particular clinical importance if invasive or potentially hazardous interventions were to be offered on the strength of a raised level. It is more important, however, that a marker of outcome is sensitive enough to allow those with a "negative" result to be discharged with minimal risk of adverse consequences. We have shown that significant neurological disability is extremely unlikely if S-100B level is low, and that this is unaffected by the existence or treatment of concurrent injuries.

In addition to a possible effect on S-100B levels, extracranial injury might also confound outcome assessment. To minimise this effect patients were asked to evaluate their disability according to their head injury alone. ${ }^{16}$ We assigned their GOSE score accordingly. Confounding factors such as extracranial injury, or indeed preinjury morbidity, would tend to increase the number of patients with a poor outcome. If that were the case, serum S-100B would appear less sensitive in identifying disability in this study - that is, the false negative rate would 
be increased. For this reason we believe that S-100B is as least as sensitive as demonstrated by these data.

\section{S-100B clearance characteristics}

The half life of S-100B in serum is quoted at around two hours in previous mild head injury work. ${ }^{25}$ This is based on analysis of concentrations in patients undergoing cardiopulmonary bypass procedures. ${ }^{26}$ The half life in this setting has more recently been estimated at around 25 minutes. ${ }^{27}$ Estimation in severely head injured patients suggests that the half life is around three hours in this group. ${ }^{28}$ Continuing release of S-100B with secondary brain injury has been shown, ${ }^{29}$ and this could account for the prolonged half life found in the severely injured. Clearance characteristics following mild head injury have yet to be confirmed. If the half life in this group is as rapid as anticipated, however, then clearly the time elapsed between the injury and the serum measurement could be expected to have a profound effect on the interpretation of the results. We chose to recruit patients within six hours of injury to minimise such effects. These data show that the S-100B concentration is extremely unlikely to fall to "normal" levels within six hours in those with prognostically significant injuries despite its rapid clearance from the serum. However, more work in this area is needed.

\section{Implications for clinical practice}

Around 500000 adult patients attend the emergency departments of England and Wales annually..$^{30}$ If our data are extrapolated to national statistics, then around 60000 (12\%) of these will be severely disabled at one month. Estimating serum S-100B level would identify those patients. Moreover, the 320000 patients $(64 \%)$ with low serum concentrations $(<0.32 \mu \mathrm{g} / \mathrm{l})$ could reasonably be discharged from follow up, once intracranial haemorrhage was excluded, with the assurance that they are likely to be able to function independently within a month.

\section{Conclusions}

This test may provide valuable prognostic information that could be used to inform clinical management decisions and, in turn, to improve outcome. The next step is to validate our derived cut off points in a second group of patients.

\section{ACKNOWLEDGEMENTS}

We thank the staff and patients of the emergency departments of Hope University Hospital, Salford; Royal Oldham Hospital; North Manchester General Hospital; Wythenshawe Hospital, Manchester; and the laboratory staff of those hospitals; the Faculty of A\&E Medicine for a research grant; the Wellcome Trust for a travel grant; Cambridge Life Sciences; Andy Vail for statistical support; Damien Bates, coordinator at Royal Oldham Hospital; and Martin Smith, coordinator at Wythenshawe Hospital.

\section{Authors' affiliations}

W J Townend, Emergency Department, Hope University Hospital, Salford, UK

M J Guy, Biochemistry Department, Hope University Hospital

B Martin, D W Yates, Emergency Department, Hope University Hospital M A Pani, Department of Internal Medicine, University Hospital, Frankfurt-am-Main, Germany

Competing interests: S-100B assay kits were provided at a reduced cost and the luminometer on which the assays were performed was supplied free of charge by Cambridge Life Sciences for the duration of the study. Byk-Sangtec Diagnostica are part sponsoring a further study in the Academic Department of Emergency Medicine, Hope Hospital.

\section{REFERENCES}

1 Jennet B, Macmillan R. Epidemiology of head injury. BM 1981;282:101-7.
2 Jennet B. Epidemiology of head injury. J Neurol Neurosurg Psychiatry 1996;60:362-9.

3 Thornhill S, Teasdale GM, Murray GD, et al. Disability in young people and adults one year after head injury: prospective cohort study. BM 2000;320:1631-5.

4 Wade DT, King NS, Wenden FJ, et al. Routine follow up after head injury: a second randomised trial. J Neurol Neurosurg Psychiatry 1998:65:177-83.

5 Ingebritsen T, Romner B, Trumpy JH. Management of minor head injury: the value of early CT and protein S-100 measurements. J Clin Neurosci 1997;4:29-33.

6 Raabe A, Grolms C, Keller M, et al. Correlation of CT findings and serum brain damage markers following severe head injury. Acta Neurochir Wien 1998;140:787-92.

7 Raabe A, Grolms C, Seifert V. S100 as a marker of outcome following severe head injury. Br J Neurosurg 1999;13:56-9.

8 Ingebrigtsen T, Romner B, Kongstad P, et al. Increased serum concentration of protein S-100 after mild head injury: a biochemical marker with prognostic value? [Letter] J Neurol Neurosurg Psychiatry 1995:59: 103-4.

9 Herrmann $M$, Curio $N$, Jost $S$, et al. Release of biochemical markers of damage to neuronal and glial brain tissue is associated with short and long term neuropsychological outcome after traumatic brain injury. $J$ Neurol Neurosurg Psychiatry 2001;70:95-100.

10 Wade DT, Crawford S, Wenden FJ, et al. Does routine follow up after head injury help? A randomised controlled trial. J Neurol Neurosurg Psychiatry 1997;62:478-84.

11 Usui A, Kato K, Abe T, et al. S100 protein in blood and urine during open heart surgery. Clin Chem 1989;35:1942-4.

12 Teasdale G, Jennett B. Assessment of coma and impaired consciousness: a practical scale. Lancet 1974; ii:81-4.

13 Sapsed-Byrne S, Gao F, Harris DNF. Neurone-specific enolase and Sangtec 100 assays during cardiac surgery. Part II. Must samples be spun within $30 \mathrm{~min}$ ? Perfusion 1997; 12:167-9.

14 Jennett B. Snoek J, Bond MR, et al. Disability after severe head injury: observations on the use of the Glasgow Outcome Scale. J Neurol Neurosurg Psychiatry 1981;44:285-93.

15 Jennett B, Bond M. Assessment of outcome after severe brain damage. A practical scale. Lancet 1975;i:480-4.

16 Wilson JT, Pettigrew LE, Teasdale GM. Structured interviews for the Glasgow Outcome Scale and the Extended Glasgow Outcome Scale. J Neurotrauma 1998;15:573-85.

17 Wilson JT, Pettigrew LE, Teasdale GM. Emotional and cognitive consequences of head injury in relation to the Glasgow outcome scale. J Neurol Neurosurg Psychiatry 2000;69:204-9

18 Levin HS, Boake C, Song J, et al. Validity and sensitivity to change of the extended Glasgow outcome scale in mild to moderate traumatic brain injury. J Neurotrauma 2001;18:575-83.

19 Altman DG. Statistics with confidence, 2nd ed. London: BM Publishing Group, 2000:1 10-16.

20 Teasdale GM, Pettigrew LE, Wilson JT, et al. Analyzing outcome of treatment of severe head injury: a review and update on advancing the use of the Glasgow Outcome Scale. J Neurotrauma 1998;15:587-97.

21 van der Naalt J, van Zomeren $\mathrm{AH}$, Sluiter WJ, et al. One year outcome in mild to moderate head injury: the predictive value of acute injury characteristics related to complaints and return to work. J Neurol Neurosurg Psychiatry 1999;66:207-13

22 Hodgkinson DW, Berry E, Yates DW. Mild head injury - a positive approach to management. Eur J Emerg Med 1994;1:9-12.

23 Romner B, Ingebrigtsen T, Kongstad P, et al. Traumatic brain damage: serum S-100 protein measurements related to neuroradiological findings. J Neurotrauma 2000;17:641-7.

24 Anderson RE, Hansson L-O, Nilsson O, et al. High serum S100B levels for trauma patient without head injuries. Neurosurgery 2001;48:1255-8.

25 Ingebrigtsen T, Waterloo $\mathrm{K}$, Jacobsen EA, et al. Traumatic brain damage in minor head injury: relation of serum S-100 protein measurements to magnetic resonance imaging an neurobehavioural outcome. Neurosurgery 1999;45:468-75.

26 Blomquist S, Johnsson $\mathrm{P}$, Lührs $\mathrm{C}$, et al. The appearance of S-100 protein in serum during and immediately after cardiopulmonary bypass surgery: a possible marker for cerebral injury. J Cardiothorac Vasc Anesth 1997;11:699-703.

27 Jönsson $\mathbf{H}$, Johnsson $\mathrm{P}$, Höglund $\mathrm{P}$, et al. Elimination of $\mathrm{S} 100 \mathrm{~B}$ and renal function after cardiac surgery. Cardiothorac Vasc Anesth 2000;14:698-701

28 Jackson RG, Samra GS, Radcliffe J, et al. The early fall in levels of S-100ß in traumatic brain injury. Clin Chem Lab Med 2000;38:1 165-7.

29 Rothoerl RD, Woertgen C, Metz C, et al. Rapid evaluation of serum S-100 levels. Case report and comparison to previous results. Brain Injury 1999;13:387-91.

30 Royal College of Surgeons. Report of the working party on the management of patients with head injuries. London: The Royal College of Surgeons, 1999. 